\title{
Atchit/V gene expression is stimulated under abiotic stresses and is spatially and temporally regulated during embryo development
}

\author{
Liliane B. de A. Gerhardt ${ }^{1}$, Cláudia Magioli ${ }^{1}$, Ana B.U.C.M. Perez ${ }^{1}$, Rogério Margis ${ }^{1,2}$, \\ Gilberto Sachetto-Martins ${ }^{1}$ and Márcia Margis-Pinheiro ${ }^{1}$ \\ ${ }^{1}$ Universidade Federal do Rio de Janeiro, Departamento de Genética, Laboratório de Genética Molecular \\ Vegetal, Rio de Janeiro, RJ, Brazil. \\ ${ }^{2}$ Universidade Federal do Rio de Janeiro, Instituto de Química, Departamento de Bioquímica, \\ Rio de Janeiro, RJ, Brazil.
}

\begin{abstract}
The expression of AtchitlV gene was analysed in Arabidopsis plants submitted to abiotic stresses. Transcript accumulation was detected in leaves in response to UV light exposure, exogenous salicylic acid administration and wounding. Transgenic Arabidopsis plants carrying Atchit/V promoter:: gus fusion also showed differential expression of the reporter gene in response to these treatments. The AtchitIV expression was also analysed during Arabidopsis embryo development. GUS assay demonstrated AtchitIV promoter activation in zygotic embryos from torpedo stage up to full maturation. Promoter deletion analysis indicated that all the 5' cis-acting elements responsible for the specific tissue expression are located in a region of $1083 \mathrm{bp}$, adjacent to the start of transcription. A negative regulatory region located between portions -1083 and -600 was also observed.
\end{abstract}

Key words: abiotic stress, Chia4 Arabidopsis thaliana endochitinase, embryogenesis, gene expression, promoter.

Received: May 12, 2003; Accepted: October 21, 2003.

\section{Introduction}

Plants respond to environmental changes by controlling the expression of a large number of genes. Endochitinases are constitutively expressed in several organs of healthy plants (Samac et al., 1990; Beerhues and Kombrink, 1994; Robinson et al., 1997; Yeboah et al., 1998). However, the modulation of chitinase expression was observed in response to Nod factors during the nodulation process (Staehelin et al. 1994 and 1995; Goormachtig et al., 1998), as well as in adverse conditions, suggesting that these enzymes are involved in plant defence responses (Samac and Shah, 1991; Margis-Pinheiro et al., 1993; Beerhues and Kombrink, 1994; Büchter et al., 1997; Busam et al., 1997; Dong and Dunstan, 1997; Yu et al., 1998; Ancillo et al., 1999). It has also been demonstrated that plant endochitinases expression is spatially and temporally regulated during plant development processes such as somatic embryogeneses (de Jong et al., 1992 and 1993). In carrots, the presence of EP3 chitinase increased the number of globular embryos of ts 11 mutants in non-permissive

Send correspondence to Márcia Margis-Pinheiro. Universidade Federal do Rio de Janeiro, Departamento de Genética, Laboratório de Genética Molecular Vegetal, Caixa Postal 68011, 21941-970, Rio de Janeiro, RJ, Brazil. E-mail: margism @ biologia.ufrj.br. temperature conditions and also promoted the transition from the globular to heart-shape stage (de Jong et al., 1992 and 1993). In Picea glauca, a chitinase gene is also activated during maturation of somatic embryos (Dong and Dunstan, 1997).

We have previously described the isolation and characterisation of A. thaliana Chia4 chitinase, the AtchitIV gene, which accumulated very rapidly in Arabidopsis leaves challenged with Xanthomonas campestris bacteria (Gerhardt et al., 1997). Recently, the expression pattern of the AtEP3 chitinase from Arabidopsis was also described. Sequence analysis revealed that $A t E P 3$ and AtchitIV correspond to the same gene (Passarinho et al., 2001). In the same study, in situ hybridization, revealed that AtchitIV/ $A t E P 3$ is expressed in "nursing" cells surrounding the embryos during the development of somatic embryos. In plants, AtchitIV/AtEP3 expression was detected in mature pollen and in growing pollen tubes until they enter the receptive synergid, but no activity was detected in embryos. AtchitIV/AtEP3 expression was also detected in hydathodes, stipules, root epidermis and emerging root hair. Based on AtchitIV/AtEP3 gene expression pattern, Passarinho and co-workers proposed that this Arabidopsis chitinase is involved in programmed cell death (PCD). 
In this paper, analyses of induction and temporal accumulation of AtchitIV transcripts in response to abiotic stresses were conducted. In order to identify the location of possible cis acting elements involved in these responses, chimeric constructs harbouring the gus coding region fused to several regions of the putative AtchitIV promoter, consisting of the intact $1923 \mathrm{bp}$ and its deletions, were used to obtain A. thaliana transgenic plants. Analyses of tissue expression pattern of the different promoter deletions during plant development were also carried out.

\section{Materials and Methods}

\section{Plant material and abiotic stress treatments}

Arabidopsis thaliana plants ecotype Columbia-0 (Lehle seeds) were grown under controlled conditions in a growth chamber at $22{ }^{\circ} \mathrm{C}$, with $75 \%$ relative humidity and at daily period of $16 \mathrm{~h}$. Five to six week-old plants were submitted to treatments and rosette leaves were collected at different times after the onset of the stresses. For salicylic acid (SA) treatment, plants were sprayed with a $2 \mathrm{mM}$ salicylic acid solution ( $\mathrm{pH} 6.5$ ), and harvested at 0,$5 ; 1 ; 3 ; 5 ; 6$ and $20 \mathrm{~h}$ after treatment. For UV irradiation treatment, plants were irradiated twice for 15 min with $254 \mathrm{~nm} \mathrm{UV}$ light at an intensity of $0,8 \mathrm{~W} / \mathrm{m}^{2}$, and harvested at $0 ; 16 ; 23$; 30; 48 and $58 \mathrm{~h}$ after exposure. For wounding stress, leaves were squeezed twice across their surface with tweezers, and harvested at 0,$5 ; 1$ and $4 \mathrm{~h}$ after wounding. Plants were submitted to heat shock treatment with a temperature of $34^{\circ} \mathrm{C}$ for $4 \mathrm{~h}$. The $5^{\circ} \mathrm{C}$ cold shock treatment was also performed for $48 \mathrm{~h}$. Transgenic plants were submitted to the same treatments.

\section{RNA-blot hybridisation}

RNA was isolated from Arabidopsis plants as described by Ragueh et al. (1989) and about $20 \mu \mathrm{g}$ of total RNA were used for northern-blot experiments. Membrane hybridisations were performed according to Sambrook et al. (1989) using the $\left[{ }^{32} \mathrm{P}\right] \alpha \mathrm{dCTP}$ AtchitIV cDNA labelled fragment at $42{ }^{\circ} \mathrm{C}$. Probes were prepared using the random priming method (Feinberg and Vogelstein, 1983). Densitometry analyses were performed using the public domain NIH ImageJ program from the National Institute of Health, US (available at http://rsb.info.nih.gov/ij/).

\section{Construction of chimeric genes}

Fusion of the $1923 \mathrm{bp}$ of the AtchitIV 5' flanking region and its deletions $(\Delta 1500, \Delta 1083, \Delta 600$ and $\Delta 300)$ to the gus coding region were obtained by introducing a $N c o$ I site at the start codon ATG of the chitinase gene by site directed mutagenesis (Ho et al., 1989; Higushi et al., 1988). To amplify the $\Delta 1923, \Delta 1500, \Delta 1083, \Delta 600$ and $\Delta 300$ fragments of the AtchitIV promoter by PCR technique, different forward primers ( 5 'cat cgt cga cga att ctt taa act aat gga aac aag ttt c3'), (5'cat cgt cga cga att caa cgg agt att tct tta cc3'),
( $5^{\prime}$ cat cet gca gaa ttc cet ggt gac ta $\left.3^{\prime}\right),\left(5^{\prime}\right.$ cat cet gca gga att cac ttt aga ttt ggt tcg act tta $\mathrm{ttt} \mathrm{a} 3^{\prime}$ ') and (5'cat cct gca gga att ccg atc ata agt cat aat tca gaa aat $\mathrm{t} 3^{\prime}$ ) were used with a general reverse primer (5'ggg agt cac cat ggt gat gtt gtt ga3'), respectively. The PCR reactions contained $100 \mathrm{ng}$ of genomic DNA, 1XPCR buffer (Perkin Elmer), $2 \mathrm{mM} \mathrm{MgCl}_{2}$, $0.2 \mathrm{mM}$ dNTPs, $5 \mu \mathrm{M}$ primer and 1 unit of Taq polymerase (Perkin Elmer). The reactions were heated to $92{ }^{\circ} \mathrm{C}$ for 5 min followed by 30 cycles of amplification consisting of $30 \mathrm{~s}$ at $92^{\circ} \mathrm{C}, 30 \mathrm{~s}$ at $60^{\circ} \mathrm{C}$, and 1 to $2 \mathrm{~min}$ at $72{ }^{\circ} \mathrm{C}$, depending on the size to be amplified. The amplified fragments were cloned into pGUS1, a plasmid with a GUS promoterless expression cassette and the 3' octopine synthase termination signal (Plant Genetic System N.V., Belgium). These fusions were cloned into the plant transformation vector pDE1001 (Plant Genetic System N.V., Belgium) using the EcoRI and HindIII restriction sites. The pDE1001 T-DNA contains the neomycin phosphotransferase gene (nptII), under the control of the NOS promoter, as plantselectable marker providing resistance to kanamicin. The 1923 bp of the 5 ' promoter region of the AtchitIV chitinase appears in the EMBL data bank under accession number Y14590.

\section{Plant transformation and histochemical GUS assay}

The plant transformation vectors were introduced into the disarmed Agrobacterium tumefaciens strain C58C1(PMP90) by triparental mating (Bevan, 1984). Arabidopsis plants obtained from seeds of ecotype C-24 (Lehle seeds) were transformed by the root explants method via $A$. tumefaciens as described by Valvekens et al. (1988) and Clarke et al. (1992). Histochemical GUS assay was performed according to Jefferson (1987) with minor modifications described by Sachetto-Martins (1995), using $1.0 \mathrm{mM}$ potassium ferri- and $0.5 \mathrm{mM}$ ferrocyanide and staining for $16 \mathrm{~h}$. Embryos were removed from seeds immersed in a $100 \mathrm{mM}$ phosphate buffer $(\mathrm{pH} 7,0)$ containing $1.8 \mathrm{mM}$ cycloheximide, and then submitted to histochemical analysis. Transgenic plants challenged with abiotic stresses were assayed for GUS $16 \mathrm{~h}$ after the onset of the stresses.

\section{Results and Discussion}

In this study we investigated the activation of the AtchitIV chitinase gene in response to abiotic stresses and during plant development. The induction of AtchitIV chitinase transcripts accumulation in leaves in response to abiotic stresses was examined by RNA blot hybridization. Five to six week-old $A$. thaliana plants were challenged with UV, salicylic acid, wounding, heat and cold shock. According to the results, accumulation of AtchitIV mRNA was induced by UV irradiation, salicylic acid and wounding (Figure 1). UV induction was evident very shortly after treatment. Maximal transcript accumulation occurred after 
$1 \mathrm{~h}$ and was maintained at high levels until $6 \mathrm{~h}$ after the onset of the stress. Despite the lower level, after $58 \mathrm{~h}$, the mRNA detected was still higher than that found in non-stressed plants (Figure 1A). Salicylic acid induced AtchitIV transcripts accumulation in A. thaliana leaves at low level, as revealed by densitometry, to be around 1,5 fold after 3,5 h of treatment (Figure 1B). The AtchitIV induction by salicylic acid, an intermediate compound of the signal transduction pathway of pathogen infection, in addition to its induction by bacteria infection (Gerhardt et al., 1997), reinforces the role of this chitinase in plant defence. In addition to UV light irradiation and SA treatment, wounding also produced AtchitIV transcript accumulation in leaves. In stressed leaves, maximal response occurred $1 \mathrm{~h}$ after wounding and transcript levels decreased rapidly afterwards (Figure 1C). In contrast to these treatments, heat and cold shock were not able to induce AtchitIV transcripts (data not shown). The PvChi4, a Phasealus vulgaris Chia 4 chitinase gene that has high identity with AtchitIV (70,2\%), is also induced by UV irradiation (Margis-Pinheiro et al., 1993). The non-activation of the AtchitIV transcript accumulation in response to heat stress contrasted to that observed on PvChi4 bean chitinase gene, which is highly induced by higher temperatures (Margis-Pinheiro et al., 1994).

A)
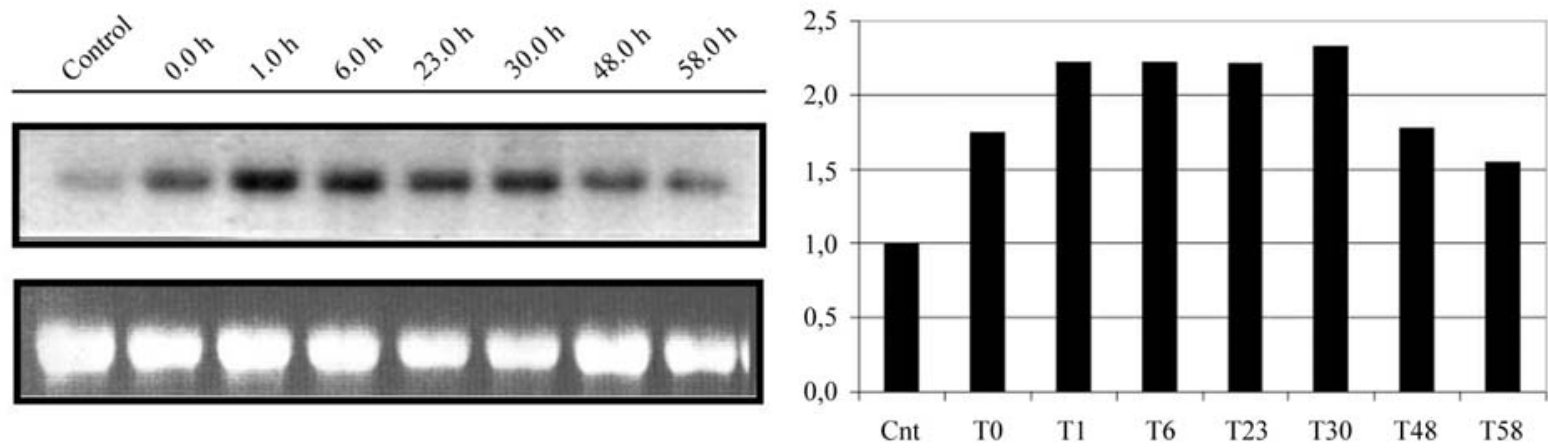

B)
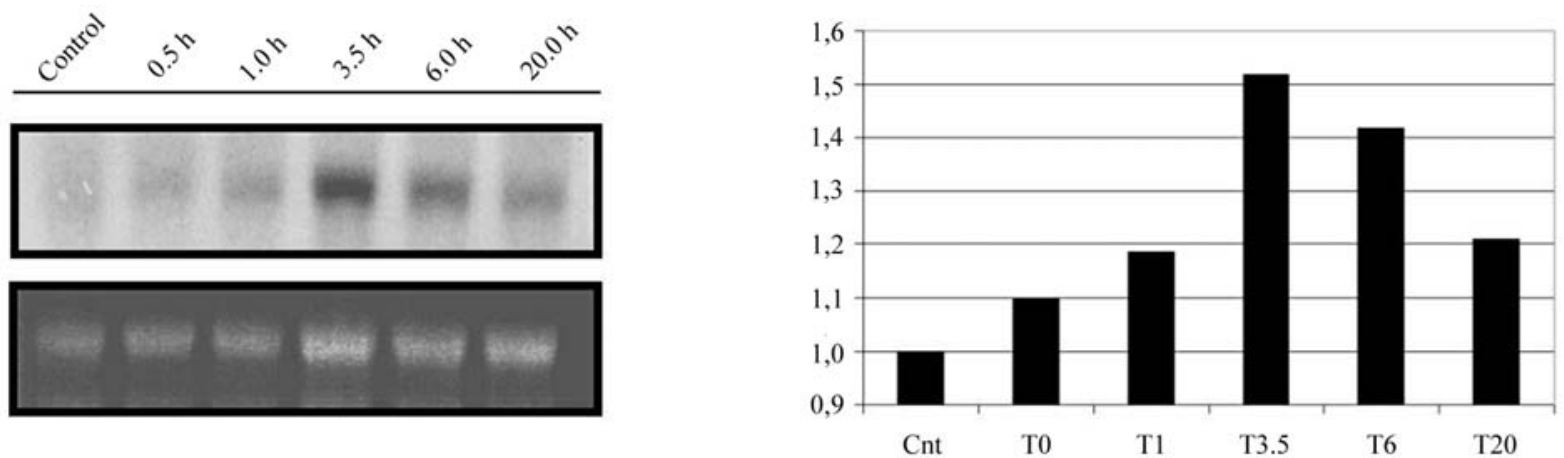

C)
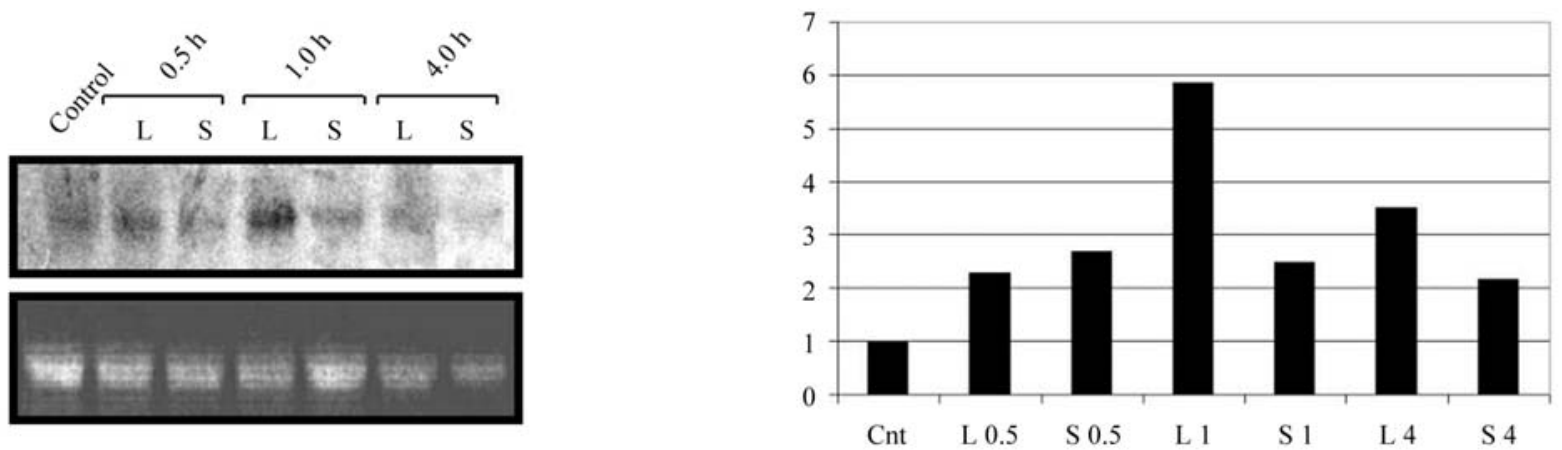

Figure 1 - AtchitIV transcripts accumulation in leaves in response to abiotic stress analysed by RNA blot hybridization. RNA from leaves of stressed plants collected at different time points, shown in hours, after the onset of treatments and RNA from leaves of control plants cultivated under normal conditions. A) UV irradiation (the 0.0 point represents the transcript levels just after the end of treatment); B) Salicylic acid treatment; C) Wounding treatment: (L) and (S) represent the local and systemic wounding leaves. The AtchitIV cDNA was used as a probe. Histograms correspond to the quantitative ratio between the autoradiographic signal and the ethidium bromide staining of RNA samples (bottom panels). 
The expression pattern of AtchitIV promoter was analysed during plant development using at least five independent GUS positive transgenic lines of each construct. The general expression pattern of the AtchitIV gene has been reported previously (Passarinho et al., 2001). In that study, the tissue expression pattern of the AtchitIV/AtEP3 gene was analysed using $1100 \mathrm{bp}$ of the promoter region fused to the gus coding region. Transgenic plants carrying this construct presented GUS activity in the meristematic region, hydathodes, root epidermis, pollen and in stigma during the fertilisation process (Passarinho et al., 2001). In order to identify possible promoter regions involved in the control of AtchitIV expression, in the present study we analysed the tissue expression pattern of transgenic plants containing promoter deletions at the positions $-1923,-1500$, $-1083,-600$ and -300 . The GUS accumulation pattern obtained was identical for the transgenic plants lines containing the $\Delta 1923, \Delta 1500$ and $\Delta 1083$ chimerical constructs, indicating that all regulatory regions needed for AtchitIV tissue expression are present within the $1083 \mathrm{bp}$ of the promoter. During seed germination, GUS staining was observed in seedling cotyledons and weakly in root, for all analysed constructs. GUS activity was also detected in the meristematic region just before the emergence of the first leaves and remained during plant development (Figure 2A). In seedlings, GUS staining was also observed in stipules, hydathodes and root epidermis (Figures 2A and 2B). In leaves of flowering plants, GUS activity was detected in hydathodes at the end of veins and along leaf margins as well (Figure 2C), confirming the constitutive expression of the AtchitIV gene in unstressed leaves of control plants (Figure 1). In flowers, GUS staining was observed in pollen, in a stage-dependent manner, and in the stigma during the fertilisation process (data not shown). These results confirm a previous study in which the expression of transgenic Arabidopsis plants containing the AtchitIV/AtEP3 1100 bp promoter was analysed (Passarinho et al., 2001). In addition, this paper reports that GUS activity was also verified in the floral receptacle, during floral senescence and at the beginning of silique development (Figure 2D). The AtchitIV activation during the senescence process of the floral receptacle corroborates the role of this chitinase in PCD proposed by Passarinho et al. (2001). During seed development, GUS activity was detected in the micropyle region (Figure $2 \mathrm{G}$ ) and in the endosperm (data not shown), next to the radicule pole, whose tissues are addressed to senescence and degradation, respectively.

In reproductive organs, transgenic plants harbouring the $\Delta 600$ and $\Delta 300$ constructs presented the same GUS activity pattern observed for the $\Delta 1923, \Delta 1500$ and $\Delta 1083$ constructs. Different from the previous constructs, plants carrying the deletions $\Delta 600$ and $\Delta 300$ expressed GUS in the whole leaves and seedlings (Figures $2 \mathrm{E}$ and $2 \mathrm{~F}$ ). These results suggest the presence of a restrictive regulatory region between the positions -1083 and -600 .
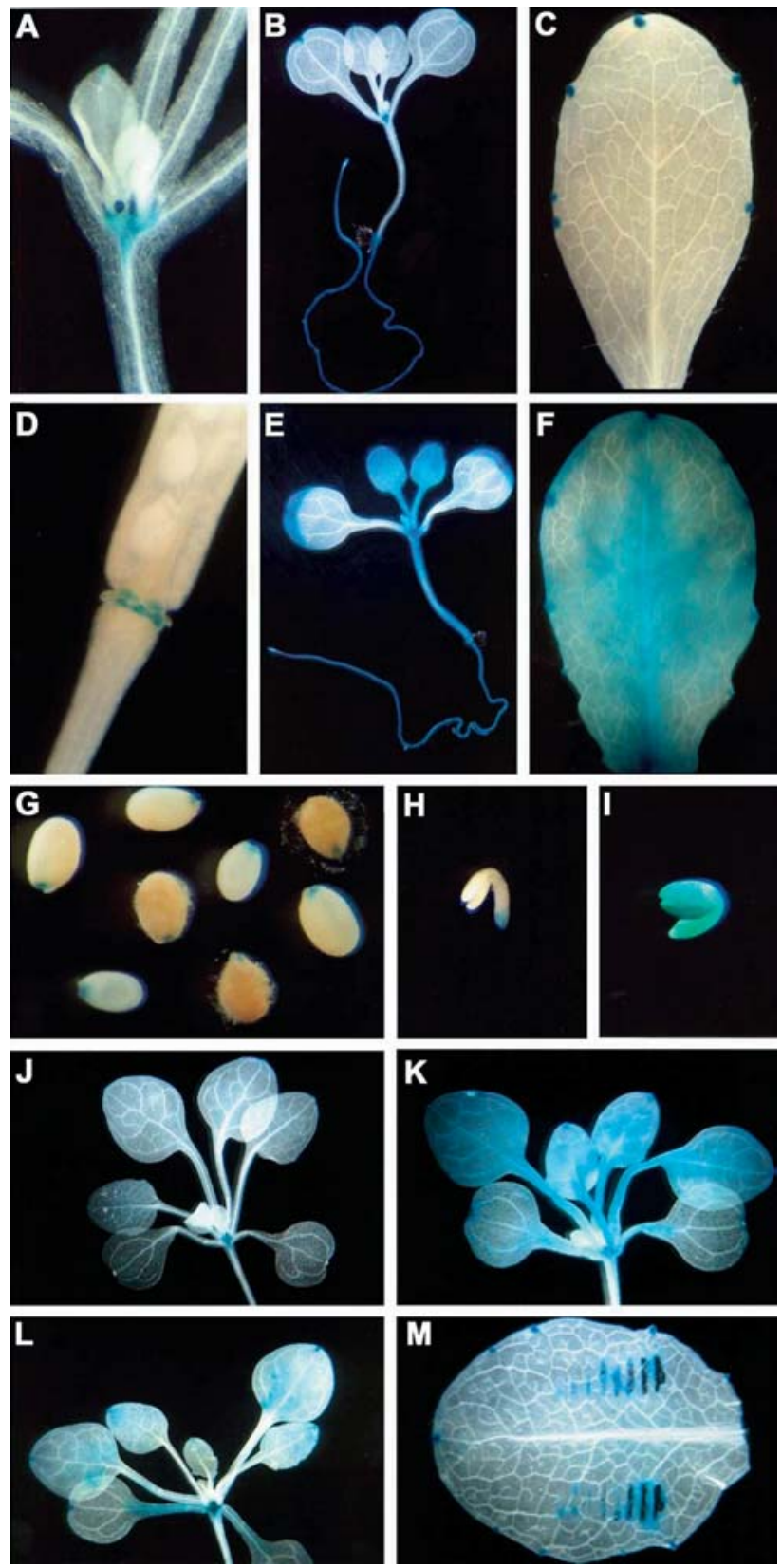

Figure 2 - Expression pattern of AtchitIV promoter in A. thaliana plants. A-D and G-M show representative GUS activity in transgenic plants harbouring promoter constructs at positions $-1923,-1500,-1083$. E and F show representative GUS activity in transgenic plants harbouring promoter constructs at positions -600 and -300 . Histochemical localisation of GUS activity in Arabidopsis transgenic plants: A) Seedling - detail of the meristematic region (40X); B) Ten day-old seedling $(6,5 \mathrm{X})$; C) Leaf (10X); D) Silique - detail of the floral receptacle (35X); E) Ten day-old seedling ( $\Delta 300$ promoter) $(6,5 \mathrm{X}) ; \mathrm{F})$ Leaf $(\Delta 300$ promoter) $(10 \mathrm{X}) ; \mathrm{G})$ Seed from different developmental stages (globular - mature stage) (40X); H) Embryo - late torpedo stage (40X); I) Embryo - mature stage (40X); J) Seventeen days-old control plant (6,5X); K) Seventeen day-old UV irradiated plant (6,5X); L) Seventeen day-old salicylic acid sprayed plant 6,5X); M) Wounded leaf (10X).

Despite some differences in the staining intensity for the $\Delta 600$ and $\Delta 300$ constructions, all five promoter:: gus fusions showed a similar tissue expression pattern during seed development. Histochemical analyses of zygotic em- 
bryos at different stages of development were performed demonstrating that the reporter gene was not expressed at the globular and heart stages (data not shown). The AtchitIV promoter activity started in the radicule pole at torpedo to late torpedo stage (Figure 2H). The same expression pattern could be observed in late torpedo embryos (data not shown). In mature embryos GUS activity was detected in the whole embryo (Figure 2I). The expression pattern observed in zygotic embryos was identical for all AtchitIV promoter deletions studied, indicating that all necessary elements to control the embryo tissue expression are present within the $300 \mathrm{bp}$. However, a more intense activity was verified for embryos containing the $\Delta 300$ bp construct, when compared to the $\Delta 1923, \Delta 1500, \Delta 1083$ and $\Delta 600$ constructs, indicating that a second regulatory negative element could be located between the positions -600 and -300 . The regulation of Chia4 chitinases during embryogenesis has been demonstrated. Picea glauca basic chitinase is involved in embryogenesis (Dong and Dunstan, 1997). Transcripts of this chitinase were highly abundant in embryogenic tissues even in cotylledonary embryos and in plantlets. Nevertheless, the carrot EP3, another Chia4 chitinase, was not detected in zygotic embryos nor in somatic embryos, although its expression had been demonstrated during seed development and somatic embryogenesis process. The EP3 expression suggests a "nursing" function during zygotic and somatic embryogenesis (Van Hengel et al., 1998). During somatic embryogenesis, the EP3 expression was verified in embryogenic and non-embryogenic cultures (Van Hengel et al., 1998). During somatic embryogenesis, Passarinho and co-workers (2001) found that AtchitIV/AtEP3 expression was restricted to embryogenic cultures in cells close to the developing embryos, but not in the embryos themselves. In contrast to our results, the expression of gus was not seen during zygotic embryo development (Passarinho et al., 2001). However, Passarinho and co-workers (2001) used the ecotype Wassilewskija to obtain Arabidopsis transgenic plants carrying AtchitIV/AtEP3 promoter-gus fusion, whereas in this study the C24 ecotype was employed for plant transformation. Differences in expression pattern between parental ecotypes of $A$. thaliana may explain the contradictory data obtained in this study and by Passarinho and co-workers concerning the AtchitIV/AtEP3 expression during embryo development.

In order to map regulatory regions in the AtchitIV promoter accounting for the response to abiotic stresses, seventeen day-old transgenic plants containing the different promoter deletions were submitted to UV irradiation and salicylic acid treatment. Both treatments were able to induce GUS staining in transgenic plants containing the $\Delta 1923, \Delta 1500$ and $\Delta 1083$ chimeric constructions (compare Figure 2J with Figures 2K and 2L). Since the basal GUS activity in leaves of plants containing the $\Delta 600$ and $\Delta 300$ deletions was already very high, it was not possible to show further accumulation of GUS activity in response to different treatments. These results suggest that the regulatory cis-elements necessary for UV light and salicylic acid response are present within the $1083 \mathrm{bp}$ of the 5' flanking region.

Wound response observed by RNA-blot hybridization (Figure1C), was also demonstrated in the promotergus transgenic lines (Figure 2M). All constructs analysed were able to mediate wound activation of the gus gene, indicating that all cis-acting elements necessary for this response are present within the $300 \mathrm{bp}$ AtchitIV proximal promoter region.

Together these results indicate a combination of positive and negative regulatory elements for the tissueexpression pattern of the AtchitIV gene. Negative regulatory elements seem to be located between -1083 and $-600 \mathrm{bp}$. These elements are responsible for expression mediated by the AtchitIV promoter to stipules, hydathodes and roots. The presence of a second negative regulatory region between -600 and -300 positions can also be suggested since a more intense widespread GUS activity was observed in $\Delta 300$ plants. Furthermore, the results presented here indicate that all 5' regulatory elements, needed for tissue expression pattern of AtchiIV gene are located within $300 \mathrm{bp}$ upstream of the translational start codon. Activation of AtchitIV promoter during stress treatments indicate that UV and salicylic acid regulatory regions are located downstream to 1083 and wounding-activating elements lie between -300 and +1 .

The induction of AtchitIV transcript accumulation in the early response to several abiotic stresses support the involvement of this chitinase in plant defence mechanism. The induction of AtchitIV transcript accumulation by salicylic acid, an intermediate in the signal transduction pathway during pathogen infection, corroborates the earlier results where this chitinase was triggered by bacteria attack (Gerhardt et al., 1997). In conclusion, we propose that AtchitIV chitinase is involved in the early responses to pathogen attacks such as the establishment of hypersensitive reaction. Besides the involvement of AtchitIV chitinase in plant defence, its expression pattern suggests an additional role in different aspects of plant development such as embryogenesis processes.

\section{Acknowledgments}

This study was supported financially by the Conselho Nacional de Desenvolvimento Científico e Tecnológico (CNPq) and FUJB. L.B. de A. Gerhardt and C. Magioli were supported by doctoral fellowships from the Coordenação de Aperfeiçoamento de Pessoal de Nível Superior (CAPES) and CNPq, respectivelly. 


\section{References}

Ancillo G, Witte B, Schmelzer E and Kombrink E (1999) A distinct member of the basic, class I chitinase gene family in potato is specifically expressed in epidermal cells. Plant Molecular Biology 39:1151.

Beerhues L and Kombrink E (1994) Primary structure and expression of mRNAs encoding basic chitinase and 1,3- $\beta$ glucanase in potato. Plant Molecular Biology 24:353-367.

Bevan M (1984) Binary Agrobacterium vectors for plant transformation. Nucleic Acids Research 12:8711-8721.

Büchter R, Stromberg A, Schmelzer E and Kombrink E (1997) Primary structure and expression of acidic, class II chitinase in potato. Plant Molecular Biology 35:749-761.

Busam G, Kassemeyer H-H and Matern U (1997) Differential expression of chitinases in Vitis vinifera L. responding to systemic acquired resistance activators or fungal challenge. Plant Physiology 115:1029-1038.

Clarke MC, Wei W and Lindsey K (1992) High-frequency transformation of Arabidopsis thaliana by Agrobacterium tumefaciens. Plant Molecular Biology Reporter 10:178-179.

de Jong A, Cordewener J, Lo Schiavo F, Terzi M, Vandekerckhove J, van Kammen A and de Vries SC (1992) A carrot somatic embryo mutant is rescued by chitinase. The Plant Cell 4:425-433.

de Jong AJ, Heidstra R, Spaink HP, Hartorg MV, Meijer EA, Hendriks T, Lo Schiavo F, Terzi M, Bisseling T and van Kammen A (1993) Rhizobium lipooligosaccharides rescue a carrot somatic embryo mutant. The Plant Cell 5:615-620.

Dong J-Z and Dunstan DI (1997) Endochitinase and $\beta-1,3-$ glucanase genes are developmentally regulated during somatic embryogenesis in Picea glauca. Planta 201:189-194.

Gerhardt LBdeA, Sachetto-Martins G, Contarini MG, Sandroni M, Ferreira RdeP, Lima VMde, Cordeiro MC, de Oliveira D and Margis-Pinheiro M (1997) Arabidopsis thaliana class IV chitinase is early induced during the interaction with Xanthomonas campestris. FEBS Letters 419:69-75.

Goormachtig S, Lievens S, van de Velde W, Van Montagu M and Holsters M (1998) Srchi13, a novel early nodulin from Sesbania rostrata, is related to acidic class III chitinases. The Plant Cell 10:905-915.

Feinberg AP and Vogelstein B (1983) A technique for radiolabelling DNA restriction endonuclease fragments to high specific activity. Anal Biochem 132:6-13.

Higushi R, Krummel RK and Saiki RK (1988) A general method of in vitro preparation and specific mutagenesis of DNA fragments: study of protein and DNA interactions. Nucleic Acids Research 16:7351-7367.

Ho SN, Hunt HD, Horton RM, Pullen JK and Pease LR (1989) Site-direct mutagenesis by overlap extension using the polymerase chain reaction. Genetics 77:51-59.

Jefferson RA (1987). Assaying chimeric genes in plants: the GUS gene fusion system. Plant Molecular Biology Reporter 5:387-405.

Margis-Pinheiro M, Martin C, Didierjean L and Burkard G (1993) Differential expression of bean chitinase genes by virus in- fection, chemical treatment and UV radiation. Plant Molecular Biology 22:659-668.

Margis-Pinheiro M, Marivet J and Burkard, G (1994) Bean class IV chitinase gene: structure, developmental expression and induction by heat stress. Plant Science 98:163-173.

Passarinho PA, Van Hengel AJ, Fransz PF and de Vries SC (2001) Expression pattern of the Arabidopsis thaliana AtEP3/ AtchitIV endochitinase gene. Planta 212:556-567.

Ragueh F, Lescure N, Roby D and Marco Y (1989) Gene expression in Nicotiana tabacum in response to compatible and incompatible isolates of Pseudomonas solonaciarum. Physiology Molecular Plant Pathology 35:23-33.

Robinson SP, Jacobs AK and Dry IB (1997) A class IV chitinase is highly expressed in grape berries during ripening. Plant Physioogy 114:771-778.

Sachetto-Martins G (1995) Isolation and characterization of glycine rich protein genes of Arabidopsis thaliana, atgrps. PhD Thesis, Universidade Federal do Rio de Janeiro, Rio de Janeiro, Brazil.

Samac DA, Hironaka CM, Yallaly PE and Shah DM (1990) Isolation and characterization of the genes encoding basic and acidic chitinase in Arabidopsis thaliana. Plant Physiology 93:907-914.

Samac DA and Shah DM (1991) Developmental and pathogeninduced activation of the Arabidopsis acidic chitinase promoter. The Plant Cell 3:1063-1072.

Sambrook J, Fritsch EF, Maniatis T (1989) Molecular cloning: a laboratory manual. 2nd edition. Cold Spring Harbor Laboratory Press, Cold Spring Harbor, NY.

Staehelin C, Granado J, Muller J, Wiemken A, Mellor RB, Felix G, Regenass M, Broughton WJ and Boller T (1994) Perception of Rhizobium nodulation factors by tomato cells and inactivation by root chitinases. Proceedings of Natural Academic of Science, USA 91:2196-2200.

Staehelin C, Schultze M, Kondorosi E and Kondorosi A (1995) Lipo-chitooligosaccharide nodulation signals from Rhyzobium meliloti induce rapid degradation by the host plant alfalfa. Plant Physiology 108:1607-1614.

Valvekens D, Van Montagu M and Van Lijsebettens M (1988) Agrobacterium tumefaciens-mediated transformation of Arabidopsis root explants using kanamicin selection. Proceedings of Natural Academic Science, USA 85:5536-5540.

Van Hengel AJ, Guzzo F, van Kammen A and de Vries SC (1998) Expression pattern of the carrot EP3 endochitinase genes in suspension cultures and in developing seeds. Plant Physiology 117:43-53.

Yeboah NA, Arahira M, Nong VH, Zhang DY, Kadokura K, Watanabe A and Fukazawa C (1998) A class III acidic endochitinase is specifically expressed in the developing seeds of soybean, Glycine max [L.] Merr. Plant Molecular Biology 36:407-415.

Yu L-X, Djebrouni M, Chamberland H, Lafontaine JG and Tabaeizadeh Z (1998) Chitinase: differential induction of gene expression and enzyme activity by drought stress in the wild, Lycopersicon chilense Dun. and cultivated, $L$. esculentum Mill. tomatoes. Plant Phyisiology 153:745-753.

Editor: Márcio de Castro da Silva Filho 